\title{
REDUÇÃO VOLUMÉTRICA PÓS-TRANSPLANTE PULMONAR UNILATERAL PARA TRATAMENTO DE HIPERINSUFLAÇÃO ENFISEMATOSA DO PULMÃO NATIVO: RELATO DE DOIS CASOS
}

\author{
After single-lung transplantation volume reduction to treat native lung emphysematous hyperinflation: \\ report of two cases
}

\begin{abstract}
Paulo Manuel Pêgo-Fernandes, Marcos Naoyuki Samano, Jader Joel Machado Junqueira, Marlova Caramoni, Ricardo Henrique de Oliveira Braga Teixeira, José Eduardo Afonso Júnior e Fabio Biscegli Jatene
\end{abstract}

\begin{abstract}
RESUMO
A hiperinsuflação do pulmão nativo é comum no pós-operatório de transplantes pulmonares unilaterais por enfisema. Quando progressiva, pode comprimir o pulmão transplantado, gerando balanço mediastinal, com prejuízo da ventilação. N estas situações, o tratamento consiste na redução volumétrica do pulmão nativo. Relatamos dois casos de hiperinsuflação, uma aguda e outra tardia de pulmão nativo, após transplante pulmonar. A mbos evoluíram com disfunção progressiva e sem resposta ao tratamento clínico. No caso agudo, optamos por cirurgia de redução de volume pul monar com uso de grampeadores e múltiplas ressecções em cunha, removendo $20 \%$ do parênquima. No caso crônico, optamos por realização de lobectomia inferior, devido ao resultado da cintilografia.
\end{abstract}

Descritores: Transplante de Pul mão, Complicações Pós-O peratórias, Enfisema Pulmonar, Redução do Volume do Pul mão, Lobectomia Pulmonar.

\section{Instituição:}

Serviço de Cirurgia Torácica do Hospital das Clínicas da Faculdade de Medicina da Universidade de São Paulo (HCFMUSP)

\section{Correspondência:}

Paulo Manuel Pêgo-Fernandes

Laboratório Anátomo-Cirúrgico do Instituto do Coração (InCor) - HCFMUSP.

R. Dr. Enéas de Carvalho Aguiar, $44-2^{\circ}$ Andar / Bloco 1 - Cerqueira César.

CEP: 05403-900 - São Paulo/SP.

Telefone/Fax: 551130695372

E-mail: paulopego@incor.usp.br

\section{INTRODUÇÃO}

0 enfisema pulmonar ocorre em aproximadamente $70 \%$ dos pacientes com doença pulmonar obstrutiva crônica (DPOC). ${ }^{1}$ Devido ao processo irreversível da doença, com redução progressiva dos fluxos expiratórios, associada à hiperinsuflação pulmonar, ${ }^{2,3}$ em estágios avançados, o transplante de pulmão torna-se a única forma de tratamento para estes pacientes. ${ }^{3,4}$

Complicações pós-operatórias como rejeição, infecção e estenose da anastomose brônquica, todas relacionadas ao pul mão transplantado, têm sido bem estudadas. ${ }^{5}$ Por outro lado, o pulmão nativo também pode ser foco de complicações agudas e tardias que afetarão a evolução do transplante. ${ }^{6,7} \mathrm{H}$ iperinsuflação de pulmão nativo, infecção, distensão de bol ha e pneumotórax são descritos como as principais destas complicações. ${ }^{7}$

A hiperinsuflação de pulmão nativo é um achado radiológico freqüente no período pós-operatório imediato e que, geralmente, se resolve dentro de alguns meses, não estando associado a uma pior evolução. ${ }^{8}$ Porém, uma hiperinsuflação progressiva pode gerar balanço mediastinal com compressão, restrição e distúrbios ventilatórios no pulmão transplantado., ${ }^{1,4}$

Relatamos dois casos de hiperinsuflação de pulmão nativo após transplante pulmonar unilateral, que cursaram com disfunção pulmonar progressiva, não responsiva a tratamento clínico, e que foram submetidos a tratamento cirúrgico com lobectomia e cirurgia de redução do volume pulmonar (CRV P). 


\section{CASO 1:}

Paciente de 41 anos, tabagista (20 anos-maço), com história de dispnéia progressiva há seis anos e diagnóstico de enfisema panlobular, com antecedente de bulectomia à direita e em tratamento no Serviço de Pneumologia do HCFM USP. A presentou progressão do enfisema, necessitando de oxigenoterapia domiciliar, até a instalação de insuficiência respiratória, com dispnéia aos mínimos esforços, classe funcional IV pela $\mathrm{New}$ York Heart A ssociation (N Y HA).

Encaminhado para avaliação de transplante pulmonar, apresentava-se em regular estado geral, emagrecido (I M C =13,4), com gastrostomia para alimentação, dispnéico e com sibilos difusos à ausculta pulmonar. Espirometria mostrava $V F^{1}=15 \%$ e CVF $=42 \%$. Cintilografia perfusional quantitativa apresentou valores de $33 \%$ e $67 \%$ nos pulmões direito e esquerdo, respectivamente. $0 \mathrm{~s}$ achados dos exames radiográficos e tomográficos eram compatíveis com enfisema bilateral grave, com perda importante de parênquima pulmonar.

A pós sete meses em lista de espera, foi submetido a transplante pulmonar direito (figura 1). Realizou-se imunossupressão com azatioprina, ciclosporina e solumedrol. 0 paciente foi encaminhado para a UTI, sendo extubado no quinto dia pósoperatório (PO), porém evoluiu com insuficiência respiratória, necessitando reintubação horas depois. Progressivamente notou-se hiperinsuflação do pulmão nativo, com desvio do mediastino e piora do padrão ventilatório. Iniciou-se ventilação independente, com utilização de cânula tipo Robertshaw, sem mel hora radiológica ou clínica.

Foi então submetido a CRVP à esquerda para suprimir o efeito compressivo sobre o pulmão transplantado, através da utilização de grampeador linear cortante e múltiplas ressecções em cunha, removendo, aproximadamente, $20 \%$ do parênquima do ápice pulmonar. Utilizou-se reforço da sutura mecânica, através da interposição de membranas de pericárdio bovino (figura 2). ${ }^{9}$

Houvemelhora radiológica, com expansão do pulmão transplantado e normalização do contorno diafragmático, além de melhora

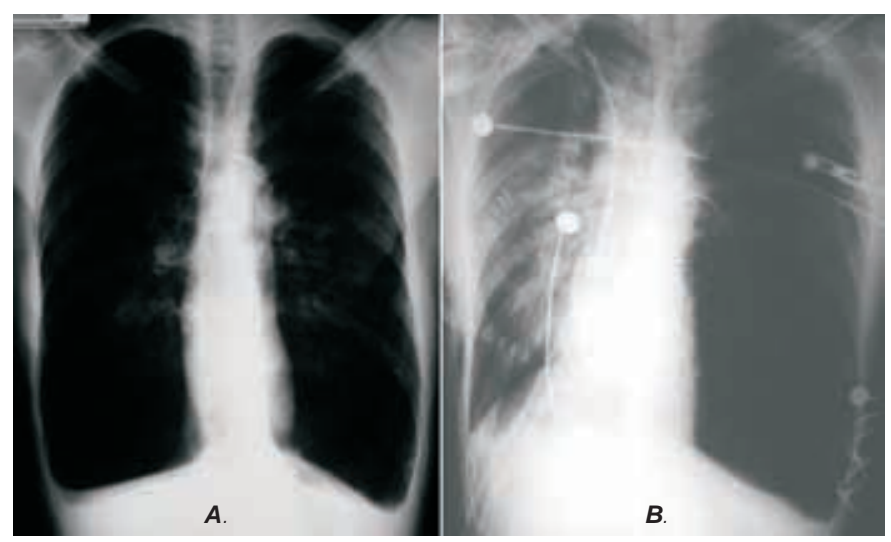

Figura 1. A. Raio-X de tórax pré-transplante, com rebaixamento e retificação diafragmática, aumento do espaço aéreo retroesternal, hipertransparência pulmonar, estreitamento da sombra cardíaca e afilamento da sombra vascular; B. Raio-X de tórax no primeiro PO de transplante pulmonar unilateral à direita, com boa expansão pulmonar e locação do dreno
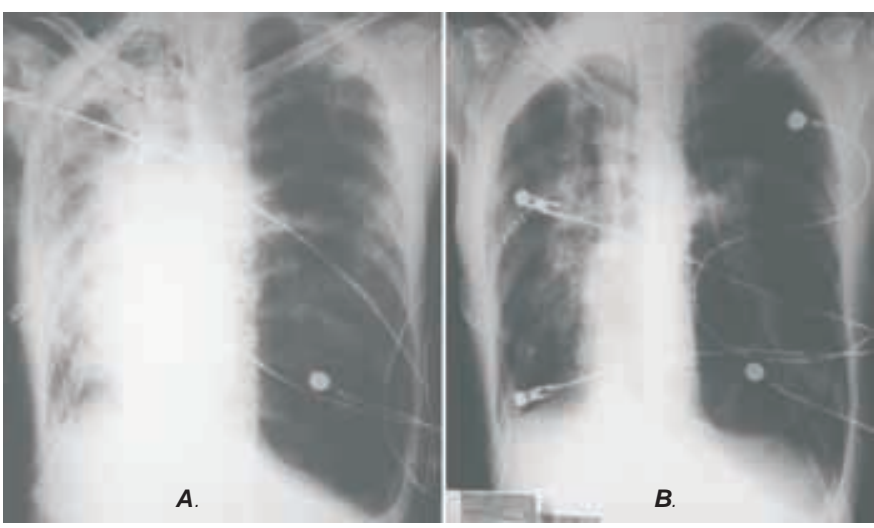

Figura 2. A. Raio-X de tórax no sétimo $P O$ de transplante pulmonar unilateral à direita, com hiperinsuflação de pulmão nativo, depressão do hemidiafragma esquerdo, deslocamento mediastinal e compressão do pulmão transplantado; B. Raio-X de tórax no primeiro PO de CRVP à esquerda, com melhora do padrão radiológico

das funções pulmonares. Porém, evoluiu com deiscência da anastomose da parede posterior do brônquio principal direito e fístula bronco-pleural. A presentou empiema e quadro séptico, evoluindo a óbito no décimo sexto PO.

\section{CASO 2:}

Paciente masculino, 46 anos, não tabagista, com dispnéia progressiva há nove anos e diagnóstico de talcose e enfisema pulmonar. Em uso de oxigenoterapia domiciliar, com consumo máximo de $0^{2}$ de $19 \mathrm{~mL} / \mathrm{kg} / \mathrm{min}$. Espirometria mostrava $V_{E F}{ }^{1}=13 \%, C V F=25 \%, C P T=110 \%$ e $V R=298 \%$. A pós dois meses em lista de espera, foi submetido a transplante pulmonar unilateral direito, sem intercorrências. A imunossupressão foi realizada com ciclosporina, azatiopina e prednisona. Houve evolução satisfatória do quadro com melhora da dispnéia, e o paciente pôde retomar suas atividades habituais.

Decorridos três anos do transplante, notou-se progressiva deterioração da função ventilatória, com sinais claros de hiperinsuflação pulmonar. Espirometria mostrava $\mathrm{VEF}^{1}=69 \%$, CV F $=71 \%, C P T=136 \%$ e V R $=278 \%$. Nesta ocasião o paciente apresentava dispnéia a pequenos esforços (figura 3).

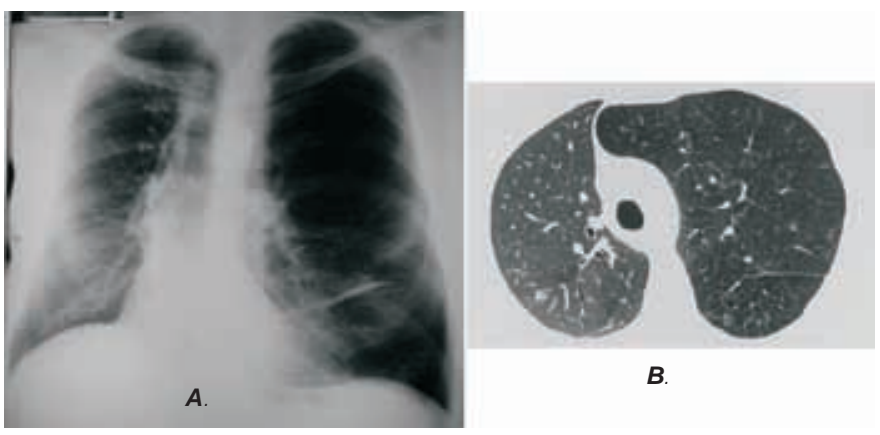

Figura 3. A.Raio-X de tórax 3 anos após transplante pulmonar unilateral à direita, com hiperinsuflação de pulmão nativo, depressão do hemidiafragma esquerdo, deslocamento mediastinal e compressão do pulmão transplantado; $\boldsymbol{B}$. TC de tórax do mesmo período 
Optou-se pela realização de redução volumétrica do pulmão através de lobectomia inferior esquerda ao invés da clássica CRV P, pois a cintilografia evidenciava menor perfusão da base pulmonar esquerda $(22 \%)$, quando comparada à direita $(78 \%)$, além do fato de que a lobectomia traria menos complicações relacionadas a fístulas aéreas, quando comparada à cirurgia redutora. N otadamente, houve mel hora dos padrões respi ratórios. Espirometria um mês após a lobectomia mostrou $V E^{1}=81 \%$, CVF $=76 \%, C P T=99 \%$ e VR $=150 \%$ e, decorrido um ano desta cirurgia, o paciente encontra-se bem (figura 4).

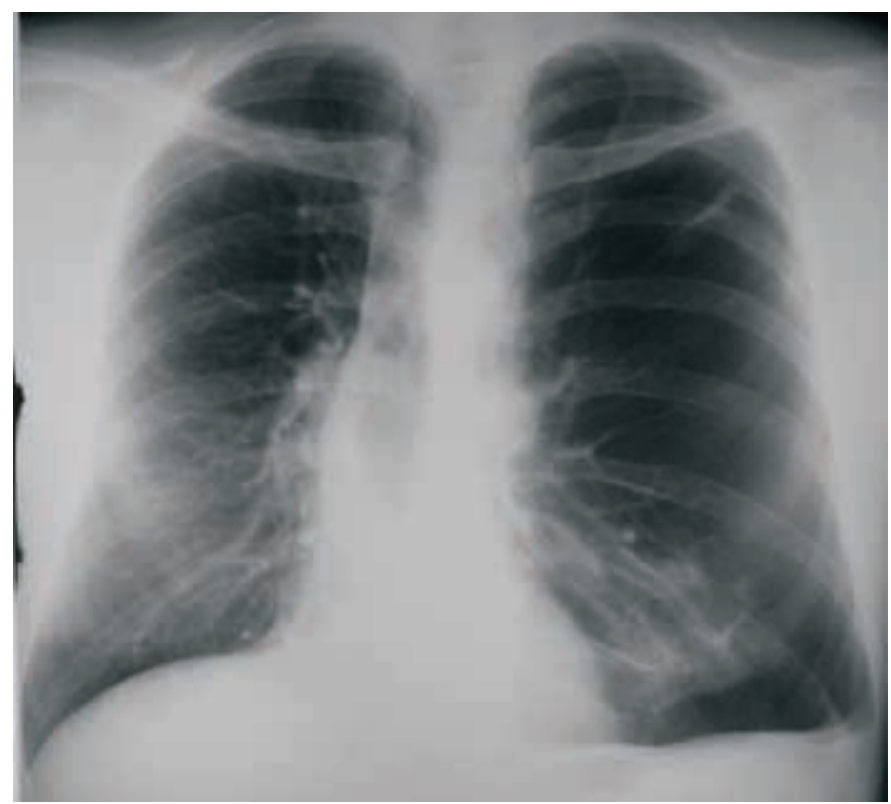

Figura 4. Raio-X de tórax controle após um ano de lobectomia inferior esquerda

\section{DISCUSSÃO}

A pesar da alta morbi/mortalidade e do risco das complicações pós-operatórias, o transplante pulmonar (TP) unilateral é uma opção terapêutica bem estabelecida para o tratamento de insuficiência respiratória resultante de enfisema. ${ }^{10}$ Possibilita utilização máxima do órgão doado (dois transplantes a partir de um único doador), permitindo uma função pulmonar satisfatória e com trauma cirúrgico rel ativamente reduzido. Porém, após o TP unilateral, a permanência de um pulmão nativo pode ocasionar a sua hiperinsuflação, com conseqüente desvio das estruturas mediastinais contralateral mente e compressão do pulmão recémtransplantado. ${ }^{6}$

A través de um estudo retrospectiv $0^{10} \mathrm{com} 27$ pacientes submetidos à TP unilateral por enfisema, podem-se definir dois principais fatores de risco que poderiam predizer a ocorrência de hiperinsuflação de pulmão nativo. 0 primeiro está ligado às condições ventilatórias do receptor - volume expiratório forçado no primeiro segundo menor que $15 \%$ e volume residual maior que o dobro do predito - são fatores de risco significantes. 0 segundo fator está ligado à uma disfunção aguda do órgão (lesão de reperfusão ou falência aguda do órgão), necessitando de ventilação mecânica prolongada com pressão expiratória final positiva (PEEP). A lém disso, pacientes com hipertensão pulmonar rel ativa têm risco aumentado de desenvolver edema pulmonar (lesão reperfusional), também necessitando de ventilação mecânica com PEEP, podendo levar a hiperinsuflação aguda de pulmão nativo.

Para critérios diagnósticos, a hiperinsuflação pulmonar aguda é definida por um conjunto de sinais radiológicos, como balanço mediastinal e retificação do diafragma ipsilateral, associado a sinais clínicos de instabilidade hemodinâmica (pressão sistólica $<90 \mathrm{mmH}$ g ou pressão arterial média $<65 \mathrm{mmHg}$, necessitando de drogas vasoativas) ou respi ratória, necessitando de uma ventilação diferenciada (inalação de óxido nítrico ou ventilação pulmonar independente) ${ }^{1,8,10}$

A diferença de complacência entre o pulmão nativo e o transplantado resulta em graves distúrbios de ventilação/ perfusão, com ventilação preferencial do pulmão nativo e perfusão do enxerto, levando a uma insuficiência respiratória e/ ou hemodinâmica no período pós-operatório. 3,8,10

Como forma de evitar este desbalanço e a morbi/mortalidade do paciente, a extubação precoce deve ser preconizada sempre que possível. A ventilação independente, com utilização de cânula tipo Robertshaw, pode ser também cogitada. Na ventilação pulmonar através de tubo orotraqueal de duplo-lúmen deve-se optar por uma ventilação mínima do pulmão nativo $(5 \mathrm{~mL} / \mathrm{kg}, 2-6 \mathrm{cpm})$. Entretanto, pela dificuldade de manutenção de cânula sel etiva bem posicionada por longos períodos em ambiente de terapia intensiva, necessitando da realização de constantes broncoscopias, sua utilização não tem sido recomendada pelo nosso grupo.

A Igumas técnicas são preconizadas na prevenção da hiperinsuflação do pulmão nativo: (1) ressecar bolhas gigantes; (2) o pulmão do doador deve apresentar capacidade vital maior que o do receptor; (3) nos casos de enfisema bilateral mente simétrico, realizar o transplante preferencial mente à direita, permitindo a expansão do pulmão nativo em direção ao diafragma, ao invés de ao mediastino; (4) extubar precocemente e evitar PEEP no pulmão nativo; (5) posicionar o paciente em decúbito lateral sobre o pulmão nativo após o transplante ${ }^{11}$.

Inúmeros estudos têm mostrado uma melhora funcional dos pacientes, com este tipo de compl icação, após al guns procedimentos cirúrgicos, como a lobectomial1 ea CRV $P^{3,3,12}$ realizadas no mesmo tempo do transplante ou posteriormente, no momento em que se identifica a hiperinsuflação. ${ }^{3}$ Essa melhora funcional pode ser evidenciada na capacidade de exercício, nos estudos de função pulmonar e na menor queixa de dispnéia.

A tualmente, tem-se descrito, com sucesso, a redução de volume pulmonar através da colocação de válvulas endobrônquicas via broncoscopia, principalmente em pacientes graves. ${ }^{13}$ Porém, apesar de se tratar de um procedimento menos invasivo, é um método ainda em fase de estudo e com resultados em longo prazo não bem estabelecidos.

A pesar do transplante pulmonar unilateral aumentar o número de operações, permitindo dois transplantes a partir de um único doador e representando uma alternativa para diminuir o número de pacientes em lista de espera, o transplante bilateral tem sido realizado com maior freqüência, como se observa no registro internacional da International Society for Heart and L ung Transplantation (ISHLT). A tualmente, cerca de $60 \%$ dos transplantes pulmonares realizados no mundo são bilaterais. ${ }^{14}$ Embora a agressão cirúrgica através da bitoracotomia ântero- 
lateral transesternal seja maior, o resultado funcional é melhor, como temos observado na prática clínica. A lém disso, a sobrevida dos transplantes bilaterais nos pacientes portadores de DPOC e enfisema por deficiência de alfa-1-antitripsina é estatisticamente maior e significativa. ${ }^{15}$

A pesar de medidas preventivas, como a realização do transplante preferencial mente à direita e a extubação precoce, a hiperinsuflação do pulmão nativo após a realização de um transplante pulmonar unilateral éuma complicação relativamentecomum e pouco previsível. Embora haja tendência na realização de transplantes bilaterais, inclusive para a DPOC, o transplante unilateral ainda é responsável por grande parte da casuística mundial e as equipes transplantadoras devem permanecer atentas a este tipo de problema.

Em nossa experiência, o resultado da redução volumétrica pulmonar foi satisfatório nas duas situações, embora o primeiro paciente tenha apresentado deiscência brônquica, empiema e sepse. Desta maneira, concluímos que este procedimento, realizado através de CRV P clássica ou lobectomia, é eficaz no tratamento desta complicação. Concluímos também que, com a tendência à realização de transplantes bilaterais e sua melhor sobrevida nos casos de DPOC e enfisema por alfa-1-antitripsina, esta complicação tenderá a diminuir gradativamente.

\section{ABSTRACT}

$\mathrm{N}$ ative lung hyperinflation is commonly seen next to single-lung transplant due to emphysema. The progressive graft compression by hyperinflation causes mediastinal shift and deterioration of the pulmonary function. U pon such situation, the surgical treatment consists in a volumetric reduction of the native lung. This work reports two cases of acute and late native lung hyperinflation after single-lung transplant with progressive pulmonary dysfunction, non-responsive to clinical treatment. In acute case, it was performed a lung volume reduction surgery with multiple wedge resections by surgical staplers, removing $20 \%$ of the pulmonary parenchyma. In chronic cases, it was performed an inferior lobectomy, because of the results of the cintilography.

Keywords: Lung Transplantation, Postoperative Complications, Pulmonary Emphysema, L ung V olume Reduction, Pneumonectomy.

\section{REFERÊNCIAS}

1. Malchow SC, McAdams HP, Palmer SM, e cols. Does Hyperexpansion of the native lung adversely affect outcome after single lung transplantation for emphysema? Preliminary findings. Acad Radiol. 1998;5(10):668-93.

2. Hodgkin JE. Prognosis in chronic obstructive pulmonary disease. Clin Chest Med. 1990;11:555-69.

3. Anderson MB, Kriett JM, Kapelanski DP, e cols. Volume reduction surgery in the native lung after single lung transplantation for emphysema. J Heart Lung Transplant. $1997 ; 16(7): 752-7$

4. Kroshus TJ, Bolman RM 3rd, Kshettry VR. Unilateral volume reduction after singlelung transplantation for emphysema. Ann Thorac Surg. 1996;62(2):363-8.

5. Murray JG, McAdams HP, Erasmus JJ, e cols. Complications of lung transplantation: radiologic findings. AJR. 1996; 166:1405-1411.

6. Venuta F, Boehler A, Rendina EA, e cols. Complications in the native lung after single lung transplantation. Eur J Cardiothorac Surg. 1999;16(1):54-8.

7. Mal H, Brugiere O, Sleiman C, e cols. Morbidity and mortality related to the native lung in single lung transplantation for emphysema. J Heart Lung Transplant. 2000; $19: 220-223.5$
8. Weill D, Torres F, Hodges TN, e cols. Acute native lung hyperinflation is not associated with poor outcomes after single lung transplant for emphysema. J Heart Lung Transplant. 1999;18(11):1080-1087.

9. Cooper JD. Technique to reduce air leaks after resection of emphysematous lung. Ann Thorac Surg. 1994;57(4):1038-9.

10. Yonan NA, el-Gamel A, Egan J, e cols. Single lung transplantation for emphysema: predictors for native lung hyperinflation. J Heart Lung Transplant. 1998;17(2):192-201.

11. Le Pimpec-Barthes F, Debrosse D, Cuenod CA, e cols. Late contralateral lobectomy after single-lung transplantation for emphysema. Ann Thorac Surg. 1996;61:231-234.

12. Kapelanski DP, Anderson MB, Kriett JM, e cols. Volume reduction of the native lung after single-lung transplantation for emphysema. J Thorac Cardiovasc Surg. 1996;111:898-899.

13. Crespo MM, Johnson BA, McCurry KR, e cols. Use of endobronchial valves for native lung hyperinflation associated with respiratory failure in a single-lung transplant recipient for emphysema. Chest. 2007;131(1):214-6.

14. International Society for Heart and Lung Transplantation. Disponível em: URL: http://www.ishlt.org/contentDocuments/lung_adult.ppt. Acessado em 21/09/06.

15. Trulock EP, Edwards LB, Taylor DO, e cols. Registry of the International Society for Heart and Lung Transplantation: Twenty-third Official Adult Lung and Heart-Lung Transplantation Report-2006. J Heart Lung Transplantation 2006;25:880-92. 